\title{
Design of Object Caching in a CORBA OTM System
}

\author{
Thomas Sandholm ${ }^{1}$, Stefan Tai ${ }^{2}$, Dirk Slama $^{1}$, and Eamon Walshe ${ }^{1}$ \\ 1 IONA Technologies plc \\ The IONA Building, Shelbourne Road, Dublin 4, Ireland \\ \{tsndhlm, dslama, ewalshe\}@iona.com \\ 2 Technische Universität Berlin \\ Sekr. E-N 7, Einsteinufer 17, D-10587 Berlin, Germany \\ stai@cs.tu-berlin.de
}

\begin{abstract}
CORBA Object Transaction Monitors (OTM) refer to a middleware technology that enable the building of transactional, objectoriented information systems running in distributed and heterogeneous environments. In this paper, we address large-scale OTM-based systems and focus attention on the important quality factors of system performance, system scalability, and system reliability. We develop an object caching strategy that employs OTM concepts such as distributed transactions and asynchronous event multicast, and show how this strategy improves an existing distributed CORBA system wrt. performance and scalability. We further describe our object caching solution as a transferable, reusable architectural abstraction, and demonstrate the application of software architectural concepts for design modeling of CORBA systems that introduce object caching.
\end{abstract}

\section{Introduction}

Software systems implemented in distributed and heterogeneous environments are becoming increasingly common as a result of the availability of communication technologies like the Internet and component technologies like distributed object middleware. This observation in particular holds for large-scale information systems, where data is distributed with software components to different nodes in a network. An important requirement here is to keep the distributed data consistent and to guarantee performance of the system.

In this paper, we focus attention on the development of a CORBA Object Transaction Monitor (OTM)-based system. CORBA OTM refers to an advanced middleware technology that has been adverted a major trend for next-generation distributed transaction processing 11]. CORBA OTM consists of the standard object request broker (ORB) providing mechanisms for remote object invocation [9], and a set of object services for distributed systems and data management, including the CORBA Object Transaction Service (OTS) [10.

We develop an object caching strategy that can be introduced to large-scale CORBA OTM-based systems [12]. The major objective is to improve system performance, while assuring system scalability and system reliability. The caching 
solution is described using the software architectural modeling concept of a connector, and its application is demonstrated with an example scenario that has been implemented using IONA's OrbixOTM product 7. The work presented has been carried out as part of the project "CORBA Object Transaction Monitor Experimentation", a cooperation between IONA Technologies Dublin and Technische Universität Berlin.

The paper is structured as follows. First, we introduce CORBA OTM and concepts relevant to improve system performance. Second, we present an object caching strategy and its implementation and test results for a sample OTMbased system. Third, we develop the software architectural connector "Object Caching with Transactional Replication" capturing interfaces and interoperation patterns of our caching solution, and show how this connector can be used to describe caching in CORBA OTM-based systems.

\section{CORBA OTM}

With the Object Management Group's (OMG) Common Object Request Broker Architecture (CORBA) 9], a standard middleware technology for the integration and interoperation of diverse software components in distributed and heterogeneous environments has been proposed. CORBA Object Transaction Monitors address enterprise computing based on CORBA, and provide additional support for security, transactions, availability, or manageability.

CORBA OTMs comprise a variety of (standard and non-standard) object management services, of which the CORBA Object Transaction Service (OTS) and the CORBA Events Service [10] are important examples. The OTS provides utilities for distributed transaction processing, i.e. for transaction management, transaction propagation, and for driving the two-phase commit protocol to coordinate different distributed resources, including databases. The Events service enables loosely coupled, asynchronous messaging between multiple event suppliers and multiple event consumers using event channels (being standard CORBA objects), based on a publish/subscribe paradigm.

CORBA OTMs can be compared to Transaction Processing (TP) Monitors of traditional client/server systems, but take the concept of a TP Monitor from procedural to open distributed object computing. A thorough treatment of enterprise computing with CORBA and CORBA OTM can be found in [14].

\section{Improving Scalability and Performance}

In large-scale distributed systems, special attention has to be paid to possible bottlenecks, due to the fact that a large number of concurrent requests have to be processed. Three main techniques can be used to circumvent this scalability and performance problem: load balancing, replication, and caching.

Load balancing involves duplicating processing in the system, e.g. by having many servers offering the same service. The main goal is to increase throughput, i.e. the number of successfully served requests, when multiple clients send 
requests concurrently. In [5], different schemes that can be used for spreading the load between servers transparently to the clients are demonstrated.

If the servers have state (manage local data), then some techniques have to be considered regarding how to replicate the data among the distributed servers [2]. Data can be replicated both to increase availability and to improve performance due to service localization. The main problem with replication is how to keep the replicas mutually consistent. Two main approaches exist here: the replicas can be updated synchronously, e.g. within a transaction for absolute consistency, or, the updates can be sent out asynchronously to trade off consistency with performance and scalability. Further, either only one replica can be updated (master/slave replication), or all replicas can be updated (peer-to-peer replication).

Object caching in a distributed environment naturally relates to load balancing and replication, but has as its main goal the improvement of performance, or user response time. Two important issues here are (a) where to locate caches, and (b) which objects to put in the caches. A list of possible cache location levels, e.g. per-process, per-node, and per-node group is presented in [18]. Many different cache location levels can coexist for the same object, as shown in 4 . The decisions taken will influence which clients can share the same objects. Shared objects must be read frequently and be updated infrequently in order for the caching to be successful.

Keeping caches accurate and consistent with the source is of predominant importance when caching objects at distributed servers. The same update approaches as with replication can be used: asynchronous, or synchronous. The asynchronous approach can be compared to optimistic locking in the database field, and must handle the case when two conflicting updates are made concurrently. Careful attention has to be paid to what information should be sent with the updates in order to minimize network traffic, while keeping the caches accurate at all times. Network traffic can also be decreased by using the multicast protocol for propagating the updates [8].

Further important issues that have to be considered when implementing an object caching strategy are object faulting and object lifetime (eviction). Object faulting concerns how to fetch the accurate value from the source and place it in the cache transparently to the client. This mechanism can, for instance, be implemented by detecting operating system page faults, as demonstrated in [8]. The problem with this approach is, however, that pages are cached, but not objects, which results in non-object-oriented trade-offs in the code. Further, such an implementation is also very operating system dependent. The object lifetime policy to choose, i.e. when to evict objects from the cache, is determined (limited) by the cache memory available. Common lifetime policies are: FIFO, TTL (Time To Live), LRU (Least Recently Used), transaction based (object lifetime equals transaction lifetime), and application server based (object lifetime equals application server lifetime) [4, 2]. 


\section{Object Caching Strategy}

\subsection{Example Scenario}

We use a geographically distributed travel agency as a sample CORBA OTMbased system, which is to be improved with an object caching strategy.

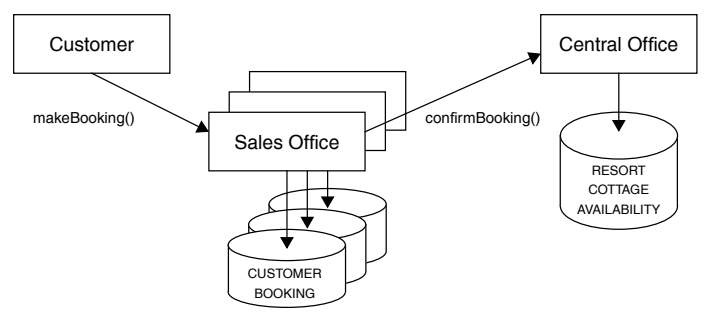

Fig. 1. A Distributed Travel Agency

The system architecture of the travel agency is shown in Fig. 1. Customers can book Cottages residing in Resorts, and browse Cottage, Resort, and cottage Availability information. Multiple SalesOffices have been introduced to decrease the load on the CentralOffice. Each booking for a cottage must first be issued on a SalesOffice; the booking is then confirmed at the CentralOffice. The Centraloffice manages persistent Resort, Cottage, and cottage Availability data in a relational database. The SalesOffice manages local Customer information, and maintains all Bookings that are made at the SalesOffice.

We assume that the system has one million Customers, and that one booking can be made each second in the system. For each booking, multiple queries on Resort, Cottage, and cottage Availability (Booking) information typically are issued. We assume that the average booking during peak system load consists of 12 queries, followed by one update (= a booking session).

\subsection{Introducing Caching}

The first decision concerns to cache Resort and Cottage objects (as these are frequently read objects, but updated infrequently) and to replicate cottage Availability data as Booking data at the distributed SalesOffices. Two different consistency policies are chosen to keep the cached objects and the replicated data consistent with the data in the Centraloffice.

Asynchronous propagation (optimistic approach) is used for keeping the cached objects accurate and consistent with the source. Asynchronous multicast propagation is selected because of performance and scalability reasons. The problem of concurrent updates here is handled when confirming bookings at the 
CentralOffice. The Centraloffice detects when an inaccurate cache has been used, and returns an exception that the booking cannot be made.

Synchronous propagation (pessimistic approach) is used for updating the replicated data (Bookings). When a booking is to be made, a distributed transaction is started at the local SalesOffice. Within this transaction, the booking is confirmed at the Centraloffice, is made persistent in the Availability table, and is replicated locally in the Booking table. The data in the two databases are hence kept synchronous by the two phase commit protocol. This approach is more time consuming, and does not scale as well as the asynchronous approach. The approach, however, assures that the local data is always consistent with the source at any point in time. This enables book keeping or invoicing tasks, for example, at the local SalesOffices without contacting the Centraloffice. We use master/slave propagation, i.e. all changes must be made at the Centraloffice first. By doing so, we avoid conflicts that can occur due to concurrently propagated updates.

Two different strategies are implemented for updating the caches, once an update event has arrived at the SalesOffice. The source can be contacted to get the currently most accurate value, or local updates can be made using object information sent with the event. Source updates are safer when concurrent updates are made. Additionally, if the original transaction aborts after the notification has been sent away, the caches will still be valid when using the source update approach, as they will read the source data in a transaction scheduled after the original one. Local updates have a significant performance advantage, though.

If an object that is not in the cache is accessed, then it is fetched from the source transparently to the clients. Once the object is fetched, it remains in the cache for the lifetime of the SalesOffice servers. When a particular Cottage is queried, the cache is filled with information for all Cottages in the same Resort. SalesOffices thus only need to cache some Resorts, which compensates the fact that no direct object eviction is implemented. Furthermore, the Availability data are decreased (and thereby also the size of the caches) as more Cottages are booked.

In order to minimize network traffic, a proper event granularity must be chosen. In our example, we use only one event channel for propagating bookings, but send object information with the events to enable updates of single cache entries (for a single object). The SalesOffices are event consumers, and the Centraloffice is the only event supplier.

The caching strategy is summarized in Table 1.

This object caching strategy has been implemented and tested by simulating the assumptions about system usage. The relation between the number of queries, and the number of updates (12 to one) is crucial to the success of the caching implementation. The more queries that are made, the more does the caching pay off. The tests were carried out by simulating both peak system load, and twice that load, in order to measure scalability of the solution. The peak system load, i.e. one booking session is started every second, was derived from 
Table 1. Caching strategy summarized

\begin{tabular}{ll}
\hline Problem & Solution \\
\hline Cached Objects & Resort and Cottage objects \\
Cache Location & Application server \\
Replica Consistency & Synchronous propagation \\
Cache Consistency & Asynchronous multicast propagation \\
Update Policy Replication & Master/Slave \\
Update Policy Caches & Update from source/local updates \\
Object Faulting & Clients access objects via application servers that \\
& transparently fetch source state \\
Cache Eviction & Application server based \\
Event Granularity & One event channel per class of objects that can be \\
& modified, events carry object level information \\
\hline
\end{tabular}

assumptions made on how the one million customers use the travel agency system. Further, the system was tested before and after the introduction of caching, and local updates were compared to updates from source. Throughput (reliability), i.e. number of successful bookings, and the response time for the bookings (performance) were measured.

A client test suite was developed for the simulations. Each client in the suite implemented a booking session as follows: (1) a query to get all Resorts was made, and one of these Resorts was picked at random; (2) all Cottages for this Resort were collected in a query, and 10 Cottages in the chosen Resort were picked randomly; (3) availability data were retrieved for each of these Cottages; (4) finally a booking was attempted for one week chosen at random from the retrieved availability data. One of these clients was started asynchronously from a shell script each second. Information on the time it took for each client to complete its booking session (user response time), and whether the booking attempt in (4) was successful was traced. Further details on test environment are available in 12. The results are depicted in Fig. 2.

The caching strategy improved reliability, scalability, and performance of the system compared to the system without caching. The caching strategy using local updates scaled better than the solution updating from source. Notable from the tests is that only half of the booking attempts were successful in twice peak system load when caching wasn't used.

The caching strategy could further be improved by redirecting clients accessing the same Resorts to the same SalesOffice. This functionality could be combined with a general load-balancing scheme to dynamically spread the load on the SalesOffice servers by using e.g. OrbixNames 7 . Also, a group of replicated CentralOffice servers could be introduced into the system, so that the CentralOffice no longer can become a bottleneck and single-point of failure. 


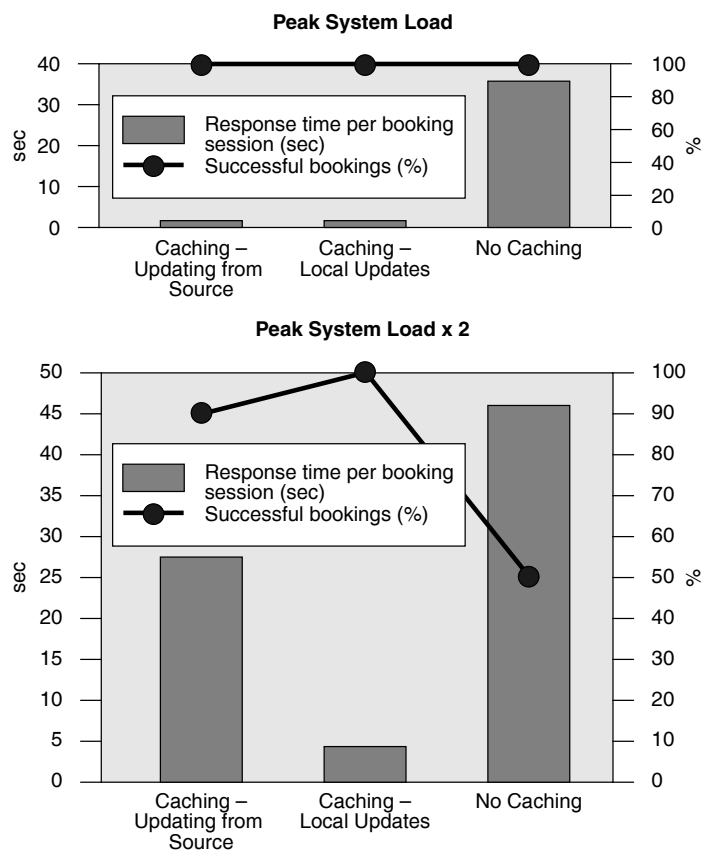

Fig. 2. Object Caching Test Results

\section{Software Architectural Design}

In the following, we present our object caching solution as a transferable, reusable connector abstraction for software architectural system design. The notion of connectors for modeling component collaborations has been mentioned in a variety of work in the area of software architecture [13], [1].

\subsection{Connector "Object Caching with Transactional Replication"}

Our particular connector concept has been proposed in [16], and has been exemplified for modeling CORBA object services in [15], [3]. Connectors are patternlike descriptions of complex component collaborations. A connector comprises the definition of roles, role interfaces, and interaction protocols.

Fig. 3 depicts the roles of our caching connector. Each role describes a collaboration responsibility, which is taken on by components in the software architecture of a particular system.

For each role, a set of role interfaces is defined (Fig. 4). These interfaces must be provided by any particular component playing the role. The role interfaces are declared using OMG IDL [9]. OID in Fig. 4 refers to a secondary identifier of an object, which is not the object reference itself, but an identifier used to 


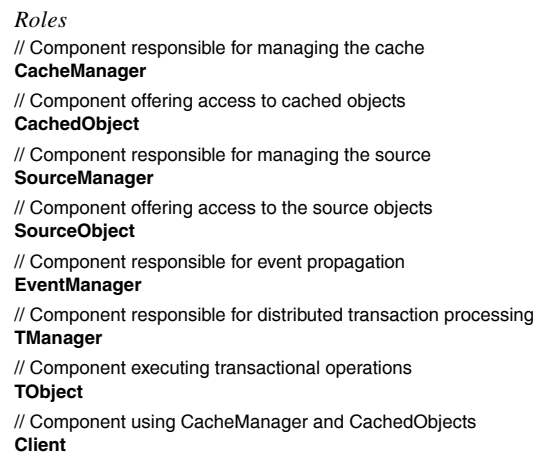

Fig. 3. Roles

map the object to a unique external entity (e.g. a primary key in a relational database).

Fig. 5 depicts the interaction protocols of our caching connector. Interaction protocols describe sequences of role interface requests along with pre- and postconditions. They are described using UML sequence diagrams [19].

The Cache Initialization and Use interaction describes the basic cache functionality. A component in role of Client sends a request for an object. If there is a valid object in the cache, it is returned directly by the component in role of CacheManager. Otherwise, the accurate state of the source object is fetched from the source. The state is used to create a cached object (cache initialization). A reference to the CachedObject is returned to the client. The next operation on the object will use the cache if it hasn't been invalidated.

The Replicated Data Modification interaction shows how the source data is kept consistent with the locally stored data. When a client wants to modify a value, a transaction is started. Within this transaction, a confirmation with the component in role of SourceManager is done, and the local database is updated. Since these operations are performed in an "all-or-nothing" fashion, the replicated data is always kept consistent. The confirmation with the SourceManager serves to detect whether other clients have updated the source concurrently, and a conflict thereby has occurred. (In our example scenario, such a conflict occurred when two clients selected the same Cottage from the cache, and then tried to book it concurrently for the same calendar week.) A conflict leads to a race condition where the first transaction to execute will succeed, and the second one will roll back.

The interaction Cache Update depicts how caches are updated by using event notifications. The events are pushed from the component in role of SourceManager to the component in role of EventManager when a SourceObject has changed. The EventManager then pushes the events to the registered Cache- 

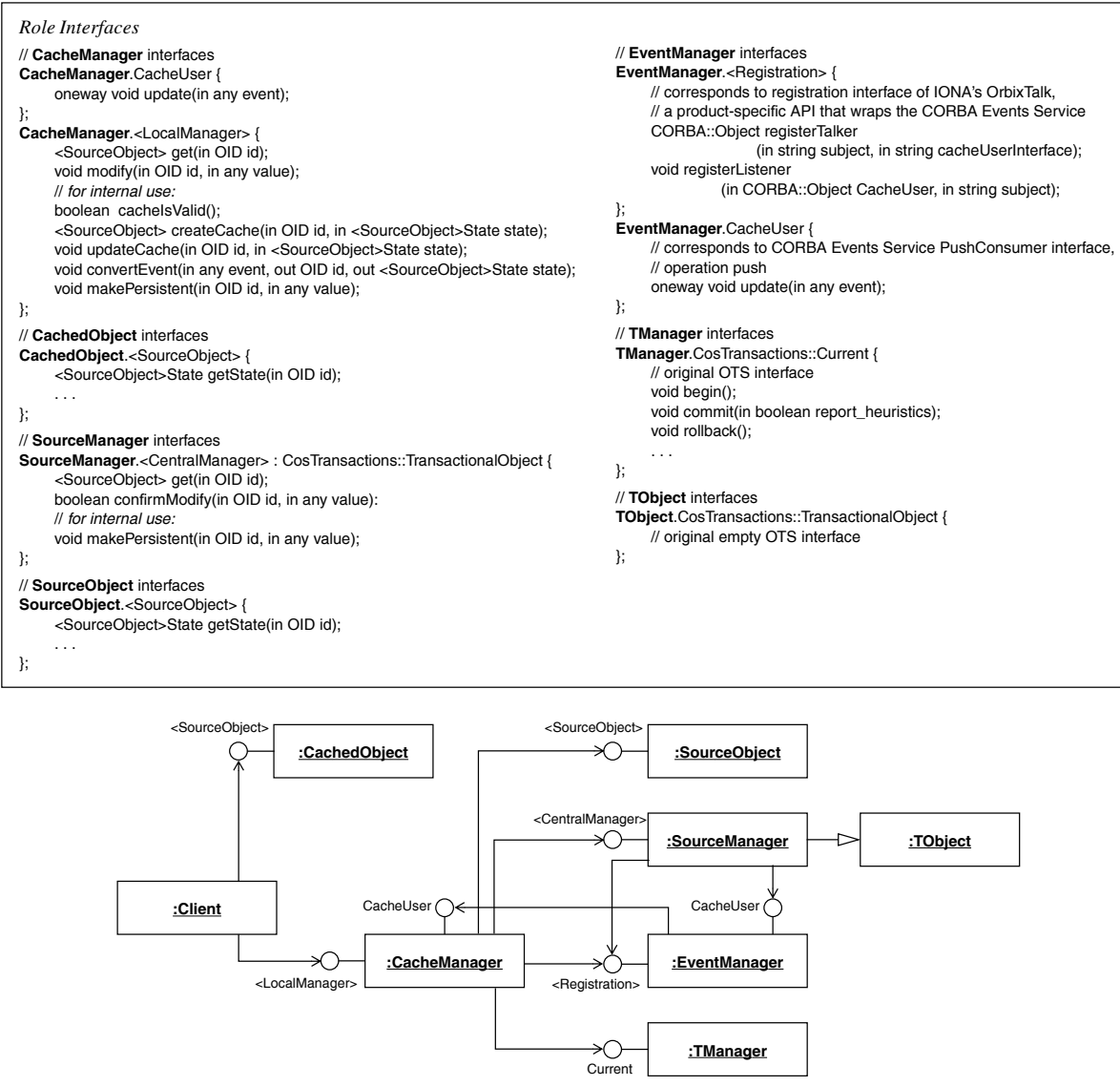

Fig. 4. Role Interfaces

Managers. The CacheManagers must filter the event to find out whether the object that has changed is in the cache. If it is in the cache, the value can be updated in two ways. First, it can be updated by getting the state from the source. Second, it can be updated locally by using the value passed by the event. The pros and cons of the two approaches were discussed in section 4.2.

\subsection{Modeling the Example Scenario}

We describe the software architecture of our example system by using the architectural framework of components, connectors, abstract architectures, and concrete architectures [17.

Components are design-time abstractions of computational system parts. Components are described using multiple views: the core functionality view, and 


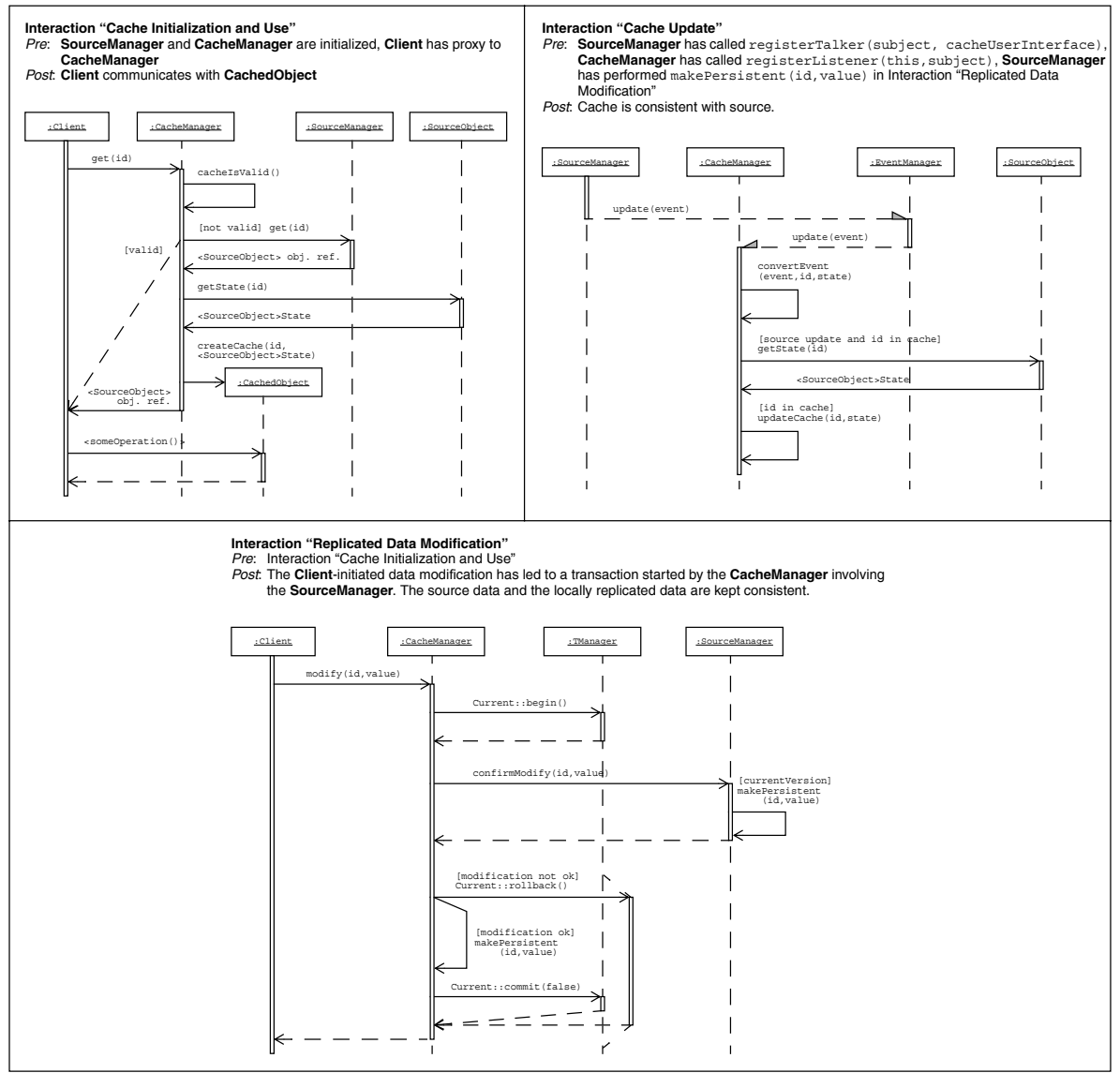

Fig. 5. Interaction Protocols

various component collaboration views. The core functionality view models the domain-oriented component features.

For our travel agency system, we can define the three application components SalesOffice, CentalOffice, and Customer, and the two service components OrbixOTS and OrbixTalk (off-the-shelf components implementing the CORBA OTS and Events Service, respectively) as design-time components. Fig. 6 depicts the exported and imported (required) system-level interfaces of the core functionality view of the SalesOffice component.

An abstract architecture of connector-based component composition is shown in Fig. 7 The components of our particular system are related by means of the generic connector "Object Caching with Transactional Replication", i.e. the connector roles are distributed to the components. This describes a requirement on the components to implement the respective role interfaces, and characterizes 


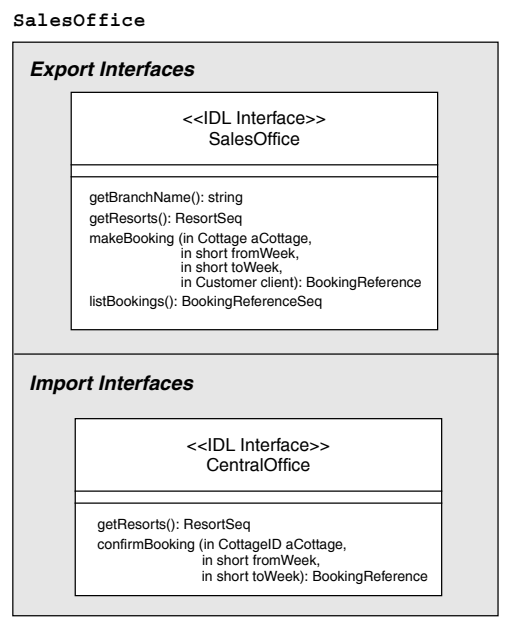

Fig. 6. Component SalesOffice - Core Functionality View

the component to interact with the other components as specified with the connector interaction protocols. Abstract architectures are software architectural descriptions on a very high level of abstraction.

Fig. 8 shows the "object caching with transactional replication" view on the SalesOffice component, i.e. the collaboration view resulting from the abstract architecture of Fig. 7]. The caching view exhibits all component features that have been introduced because of the caching rationale (as opposed to the core functionality view). The caching functionality is now exposed with new exported and imported interfaces, such as the provided CacheUser interface, or the required OTS Current interface to start, commit and abort distributed transactions. The SalesOffice component now imports and exports the Resort and Cottage interfaces unchanged.

Fig. 8 also shows the SalesOffice component's representation and export and import representation-map 1 , i.e. the internal realization design and programlevel interfaces of the component. This diagram describes implementation details of the component as a distributed, transactional CORBA server, and is expressed using UML class modeling. In the representation part, we can e.g. see that the cache is structured in a hierarchical containment tree. The Sales $\mathrm{ff}$ ice contains a collection of cached Resorts, and each cached Resort contains a collection of cached Cottages.

The set of all component descriptions of the same (object caching) view is called a concrete architecture to an abstract architecture. The software architecture of a particular system is thus described on two different levels of abstraction.

${ }^{1}$ We adapted the terminology of representation and representation-map from the ACME ADL [6]. 


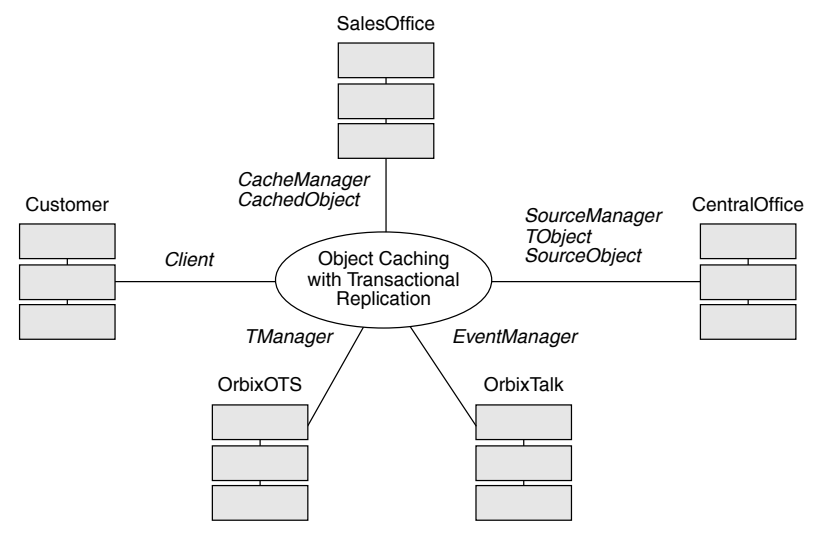

Fig. 7. Abstract Architecture of Travel Agency System

Overall, the architectural framework of design-time components, connectors, abstract and concrete architectures employs a clear separation of modeling concerns, and enables a pattern-oriented, structured approach to architectural software system representation.

\section{Conclusion}

In this paper, we developed an object caching strategy for CORBA OTM-based systems which addresses system reliability, system scalability, and, in particular, system performance. We demonstrated object caching for a sample distributed, transactional CORBA system, and showed how the caching solution proposed increased system performance notably. We abstracted the caching functionality and interoperation patterns into a software architectural connector, which was used to model the complex component collaborations of our example system, and also serves as a reusable design solution to object caching that can be applied to other CORBA OTM-based systems.

The caching solution can be summarized as follows:

Reliability of the solution was assured by asynchronous updates of the caches, and by synchronous modification of the replicated data. In terms of our connector, this behavior is captured as follows: the SourceManager sends an update event through the EventManager to the CacheManagers (interaction protocol "Cache Update"), and data modified in the database of the SourceManager is replicated in the CacheManager's database within a distributed transaction started by the CacheManager (interaction protocol "Replicated Data Modification").

Scalability was improved by service localization. All queries can be performed locally because of the caches, which thereby improve load balancing. This is captured in the connector interaction protocol "Cache Initialization and Use". The 

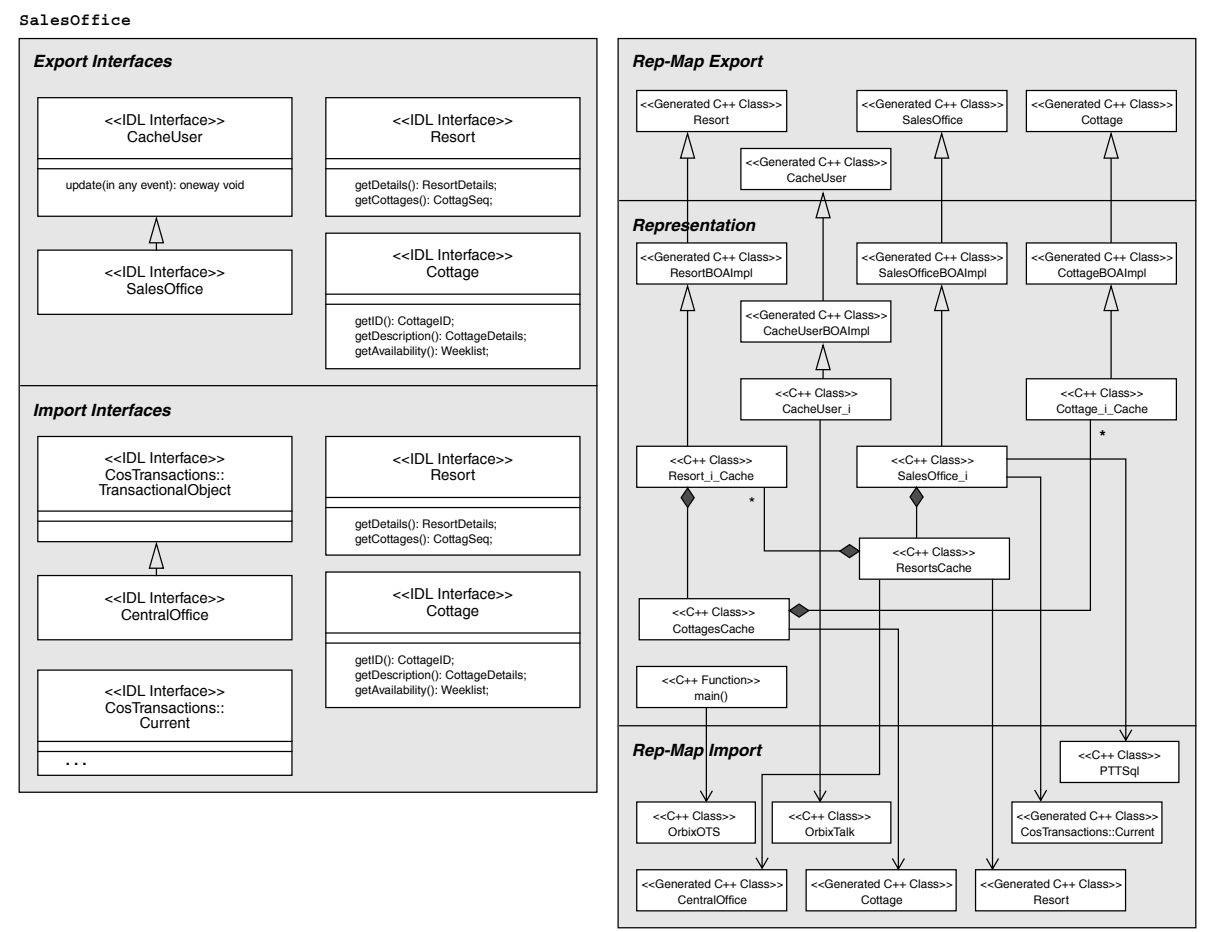

Fig. 8. Component SalesOffice - Object Caching with Transactional Replication View

CachedObject offers the same interface as the SourceObject, and the CacheManager is responsible for localizing the SourceObject, i.e. converts the SourceObject into a CachedObject. Service localization is also achieved by data replication, as mentioned previously.

Performance of the system was notably improved through the caches, and has been addressed in line with system scalability and system reliability. The design decisions regarding performance are hence documented in all three interaction protocols of our caching connector.

Acknowledgements. We would like to thank Prof. Herbert Weber, TU Berlin, and Fiona Hayes, IONA Technologies Dublin, for their continuous project support. We would also like to thank Prof. Janis Bubenko, University of Stockholm, for co-supervising the thesis underlying this paper.

\section{References}

1. L. Bass, P. Clements, R. Kazman. Software Architecture in Practice. AddisonWesley, 1998. 
2. P. Bernstein, E. Newcomer. Principles of Transaction Processing. Morgan Kaufman, 1997.

3. S. Busse, S. Tai. Software Architectural Modeling of the CORBA Object Transaction Service. In Proc. COMPSAC'98, IEEE Computer Society, 1998.

4. A. Chankhunthod, P.B. Danzig, C. Neerdales, M.F. Schwartz, K.J. Worrel. A Hierarchical Object Cache. Technical Report, CU-CS-766-95. University of Colorado, 1994.

5. R. Friedman, D. Mosse. Load Balancing Schemes for High-Throughput Distributed Fault-Tolerant Servers. Technical Report, TR96-1616, Cornell University, 1996.

6. D. Garlan, R. Monroe, D. Wile. Acme: An Architecture Description Interchange Language. In Proc. CASCON97, 1997.

7. IONA Technologies. OrbixOTM Guide. IONA Technologies plc., 1998.

8. R. Kordale, M. Ahmad. Object Caching in a CORBA compliant System. Technical Report, GIT-CC-95-23, Georgia Institute of Technology, 1995.

9. Object Management Group. The Common Object Request Broker: Architecture and Specification, rev.2.2. OMG, 1998. On-line at http://www.omg.org

10. Object Management Group. CORBAServices: Common Object Services Specification. OMG, 1997. On-line at http://www.omg.org

11. R. Orfali, D. Harkey. Client/Server Programming with Java and Corba, 2nd edition. Wiley, 1998.

12. T. Sandholm. Object Caching in a Transactional, Object-Relational CORBA Environment. Masters Thesis, University of Stockholm, 1998.

13. M. Shaw, D. Garlan. Software Architecture: Perspectives on an Emerging Discipline. Prentice-Hall, 1996.

14. D. Slama, J. Garbis, P. Russell. Enterprise CORBA. Prentice-Hall, 1999.

15. S. Tai, S. Busse. Connectors for Modeling Object Relations in CORBA-based Systems. In Proc. TOOLS 24, IEEE Computer Society, 1997.

16. S. Tai. A Connector Model for Object-Oriented Component Integration. In Proc. ICSE'98 Workshop on Component-Based Software Engineering, 1998.

17. S. Tai. Constructing Distributed Component Architectures in Continuous Software Engineering. PhD Thesis, TU Berlin, 1999. to appear.

18. D. Terry. Distributed Name Servers: Naming and Caching in Large Distributed Computing Environments. Technical Report, CSD-85-228. University of California, Berkeley, 1985.

19. UML Partners. The Unified Modeling Language, v1.1. OMG, 1997. On-line at http://www.omg.org 